\title{
Síndrome de infusión por propofol en el adulto
}

\section{Propofol infusion syndrome}

Bruno Caracci ${ }^{1}$, Fernando Aranda ${ }^{1}$

\begin{abstract}
Propofol infusion syndrome is a rare but lethal complication of propofol administration. The most common clinical presentation is high anion-gap metabolic acidosis, heart failure, rhabdomyolysis and hyperkalemia in patients receiving a prolonged propofol infusion. It has an estimated incidence of $1,1 \%$ in patients receiving propofol and its mortality varies between 18 and $81 \%$. The most important risk factor is an infusion over $4 \mathrm{mg} / \mathrm{kg} / \mathrm{h}$ of propofol and a critically sick patient. Management of a diagnosed PRIS is based on immediate discontinuation of propofol and support therapy.
\end{abstract}

\section{Resumen}

El síndrome de infusión de propofol es una complicación rara, pero posiblemente letal de la administración de propofol (Propofol Infusion Syndrome, PRIS) Generalmente, se presenta como una acidosis metabólica con anion Gap aumentado, falla cardiaca rápidamente progresiva, rabdomiólisis e hiperkalemia, junto con el antecedente claro de una infusión prolongada de la droga. La incidencia se estima en un 1,1\% en los pacientes que reciben propofol y su mortalidad varía entre el 18 al $81 \%$. Los factores de riesgo son: una infusión de propofol mayor a $4 \mathrm{mg} / \mathrm{kg} / \mathrm{h}$ y pacientes críticos. El tratamiento se basa en la suspensión de la droga y medidas de soporte hemodinámico.
Key words: Propofol, Propofol infusion syndrome, Anesthetics

\section{Palabras clave:}

Propofol,

Síndrome de infusión de propofol, Anestésicos

Médico anestesiólogo, Hospital Gustavo Friecke, Universidad de Valparaíso.

Fecha de recepción: 20 de marzo de 2018

Fecha de aceptación: 11 de junio de 2018

\section{ORCID}

https://orcid.org/0000-0002-9138-5326

\section{Correspondencia:}

Dr. Bruno Caracci

Email: brunocaracci@gmail.com 


\section{Introducción}

propofol (2, 6-diisopropilfenol) es un fármaco hipnótico-sedante usado comúnmente para inducir la anestesia general y sedación en la Unidad de Cuidados Intensivos (UCI)[1].

Una complicación rara, pero potencialmente fatal, es el denominado síndrome de infusión de propofol (Propofol Infusion Syndrome, PRIS). Si bien no existe una definición ampliamente aceptada, la clínica reportada, generalmente, consiste en una acidosis metabólica con anion Gap aumentado que no se explica por otra etiología, arritmias cardiacas (especialmente de tipo Brugada), falla cardiaca rápidamente progresiva, rabdomiólisis e hiperkalemia, junto con el antecedente claro de una infusión prolongada de la droga[1].

Los primeros casos fueron descritos en pacientes pediátricos a principios de la década de 1990[3], con reportes en adultos publicados posteriormente (4).

Debido a esto, la administración de alimentos y medicamentos de los Estados Unidos (Food and Drug Administration (FDA)), publicó una advertencia contra el uso de propofol en infusiones continuas, siendo actualizada en 2006 para informar una dosis máxima de $4 \mathrm{mg} / \mathrm{kg} / \mathrm{h}[5]$. A su vez, la Agencia de Medicamentos Europea (European Medicines Agency (EMA)), recomienda la búsqueda activa de signos sugerentes de PRIS en pacientes con infusiones prolongadas de propofol[6].

\section{Epidemiología}

La incidencia real del PRIS es desconocida y la mayoría de la evidencia surge de reportes de casos aislados. Sumado a esto, la falta de criterios diagnósticos unificados hace que los datos clínicos disponibles sean extremadamente heterogéneos[7].

En un trabajo publicado por Hwang et al., estimó la incidencia en pacientes con Status Epiléptico Refractario de PRIS en $17 \%$ para pacientes que recibían al menos $5 \mathrm{mg} / \mathrm{kg} / \mathrm{h}$ y $31 \%$ en los que recibían más de $6 \mathrm{mg} / \mathrm{kg} / \mathrm{min}[8]$.

Un estudio multicéntrico en 11 instituciones de Estados Unidos de Roberts et al., siguió a pacientes que recibieron una infusión de propofol por más de 24 h y encontró una incidencia de PRIS de 1,1\% (11 de 1.017$)$, con una mortalidad de $18 \%$. El síndrome se presentó en promedio al tercer día de infusión y los pacientes que lo desarrollaron presentaban mayor puntuación en el APACHE[9].

Se han reportado mortalidades de hasta un $81 \%$, dependiendo de la definición de PRIS[7]. En una revisión de Krajcová et al., de 153 casos publicados en la literatura encontró una mortalidad general de 51\%, con una tendencia a la disminución $(74 \%$ previo a $2001,64 \%$ en el periodo $2001-2006$ y $34 \%$ en casos publicados luego de ese período). En un $90 \%$ de los casos el síndrome se desarrolló como parte de sedación en UCl y sólo en un 10\% de los casos el síndrome apareció como parte de una anestesia[1].

\section{Factores de riesgo}

Dentro de los factores de riesgo para el desarrollo de PRIS se encuentra, en primer lugar, el antecedente claro de una infusión continua de propofol. Sumado a esto existen factores relacionados con el paciente y condiciones concomitantes que podrían aumentar la incidencia o mortalidad del PRIS. (Tabla 1).

\section{Infusión de propofol}

En la revisión de Krajcová se identificaron como principales factores de riesgo para mortalidad la duración de la infusión (sobre 20 horas OR 43,5 [9,9$191,4] p<0,001$ ), la dosis promedio por hora (sobre $5 \mathrm{mg} / \mathrm{kg} / \mathrm{h}$ OR $4,2[1,98-8,9] \mathrm{p}<0,001)$ y la dosis acumulativa sobre $360 \mathrm{mg} / \mathrm{kg}$ (OR 2,74 [1,33-5,68)[1].

Sin embargo, en el estudio publicado por Roberts et al., se reportó que se encontró sólo un $18 \%$ de los pacientes que desarrolló PRIS utilizó dosis mayores a $5 \mathrm{mg} / \mathrm{kg} / \mathrm{h}$, por lo que no sería requisito para el desarrollo del síndrome una infusión elevada.

Por último, existe a la fecha un reporte de PRIS en una paciente de 29 años con una dosis única de 100 $\mathrm{mg}$ propofol para un legrado uterino[10].

\section{Clínica}

Tanto la acidosis metabólica $(2,48[1,13-5,45] p$ $<0,02)$, arritmias (OR 4,6 [2,19-9,55], p < 0,001), hiperkalemia (OR 2,86 $[1,29-6,34] p<0,009)$ y fiebre (OR $8,25[2,71-25,15] \mathrm{p}<0,001)$ serían factores independientes de mortalidad en el PRIS[1].

\section{Enfermedad crítica y déficit de carbohidratos}

Se ha postulado que el propofol sería un gatillante del síndrome en pacientes que se encuentran en estado crítico, debido a que el estrés ocasionado por la patología aumenta los niveles sistémicos de catecolaminas y esteroides. Aparentemente la injuria traumática aguda sería un factor de riesgo en sí mismo[1]. Además, los pacientes con déficit de carnitina[11], enfermedad mitocondrial subclínica[12] que poseen depósitos disminuidos de glicógeno (niños, malnutrición 


\begin{tabular}{ll} 
& Tabla 1. Factores de riesgo para desarrollar PRIS \\
\hline Infusión de propofol & Duración: Sobre 48 horas \\
- & Dosis horaria: \\
& - Sobre $4 \mathrm{mg} / \mathrm{kg} / \mathrm{h}$ \\
- & Dosis Total: Sobre $360 \mathrm{mg} / \mathrm{kg}$ \\
- Niños & - Enfermos críticos \\
Paciente & - Especialmente lesión cerebral traumática \\
- Déficit de carbohidratos & - Niños \\
- Malnutrición crónica \\
- Ancianos \\
Tratamiento
\end{tabular}

severa, ancianos crónicamente enfermos), dependen aún más del metabolismo lipídico, el cual se encuentra comprometido en el PRIS[13] (ver fisiopatología).

\section{Fisiopatología}

\section{Toxicidad mitocondrial}

La fisiopatología del PRIS aún no se dilucida. Debido a la similitud estructural del propofol con la coenzima Q se postuló inicialmente que el mecanismo era el desacoplamiento de la cadena transportadora de electrones mitocondrial[1]. Se realizaron una serie de experimentos en animales en donde se encontró una relación lineal entre la concentración de propofol y la capacidad respiratoria en mitocondrias aisladas de rata[1],[14].

En un trabajo publicado recientemente por Vanlander et al., demostró que el propofol inhibe la actividad conjunta del citocromo II+III en mitocondrias de rata anestesiadas con dosis crecientes de propofol. Sin embargo, se observó que la actividad por separado de cada citocromo se mantenía inalterada, lo que sugiere la inhibición de la coenzima Q (transportador de electrones entre complejo II y III) (Figura 1)[15].

En humanos que han fallecido por PRIS se ha observado una disminución de la actividad de los complejos respiratorios, sin una disminución de su concentración[15].

\section{Inhibición de la oxidación de ácidos grasos}

Otra hipótesis sugiere que el propofol inhibiría la oxidación de ácidos grasos. Esto proviene de la observación de que niños con PRIS presentaban concentra- ciones de derivados de la acylcarnitina elevadas que se normalizaban luego de detener la infusión de la droga[16].

La enfermedad crítica ocasiona liberación de catecolaminas, activando la lipólisis endógena, lo que aumenta la acumulación de ácidos grasos. Sumado a esto, una dosis de propofol de $4 \mathrm{mg} / \mathrm{kg} / \mathrm{h}$ proporciona $2-3$ g/kg de lípidos cada 24 h. En niños, esto es equivalente a una carga de grasas aportada por una nutrición parenteral total[17]. Debido a que la mayoría de los casos de PRIS se asocian a dosis superiores a este límite es posible que el vehículo lipídico provoque una carga excesiva de lípidos en un paciente con su metabolismo disminuido[18].

\section{Injuria muscular cardiaca y periférica}

Un hallazgo común en pacientes que reciben propofol en infusión prolongada es la elevación de la CK y troponinas[17].

Como ya se ha dicho anteriormente, el propofol inhibe la fosforilación oxidativa, reduciendo la utilización de oxígeno y el transporte mitocondrial de electrones y la utilización de ácidos grasos, produciendo un desbalance entre aporte y gasto energético que afecta particularmente al músculo cardiaco y estriado.

Además, se ha observado una inhibición de los receptores beta adrenérgicos[19] y una acción directa en los canales de calcio voltaje dependiente[20], lo que resulta en contractibilidad cardiaca disminuida y una disminución de la respuesta a catecolaminas.

Finalmente, se ha reportado degeneración miofibrilar en músculo cardiaco y periférico asociada a niveles elevados de catecolaminas exógenas y endógenas[21]. 


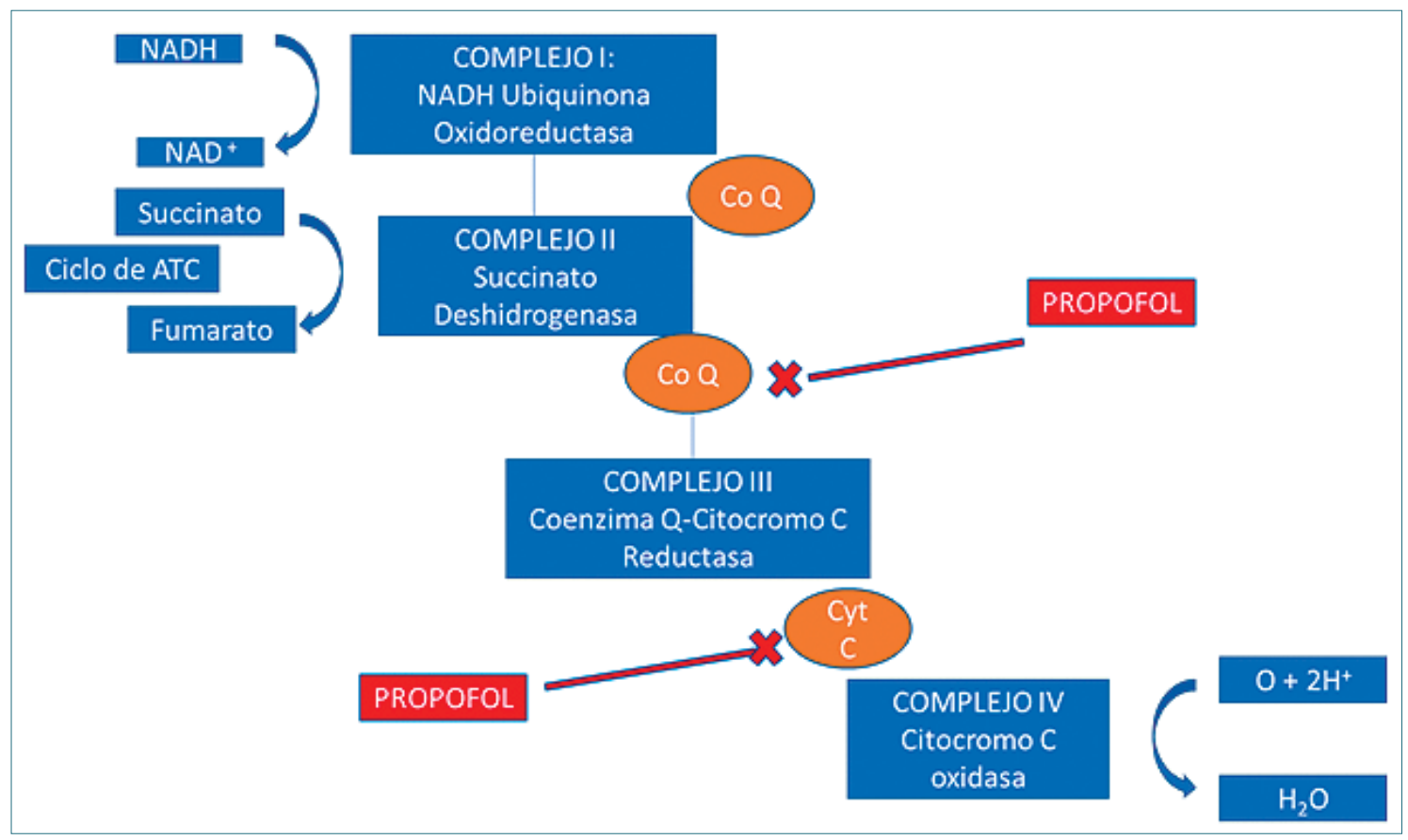

Figura 1. Las acciones inhibitorias del propofol en la cadena trasportadora de electrones se producen principalmente por una inhibición de la coenzima Q en el complejo II y del citocromo C en el complejo IV, produciendo una falla en la producción aeróbica de ATP. CoQ: Coenzima Q, Cyt C: Citocromo C. Adaptado de referencia 17.

\section{El rol de los esteroides}

Se estima que los corticoides tendrían un rol en gatillar la patología, pero no es su progresión o gravedad[17]. No existe evidencia clara sobre el mecanismo de daño de los corticoides. Una de las teorías es que se asociarían a daño miofibrilar en el músculo cardiaco y estriado[22]. Este mecanismo fisiopatológico ha sido ampliamente estudiado en el uso crónico de corticoides, pero no existe evidencia clara de si el uso agudo tendría algún rol en la miopatía asociada a propofol[17].

\section{Ciclo catecolaminas-propofol}

El uso de catecolaminas y corticoides exógenos se ha descrito como un factor de riesgo para el desarrollo de PRIS[17].

En un modelo experimental ovino, los animales fueron sometidos a una infusión de catecolaminas (epinefrina, norepinefrina y dopamina) y propofol. Las catecolaminas incrementaron significativamente el gasto cardiaco, disminuyendo la concentración plasmática de propofol, lo que se asociaba con reversión de la anestesia. Debido a esto, los animales requirieron dosis crecientes de propofol para mantener un nivel adecuado a medida que se aumentaba la infusión de catecolaminas[23].

Es por esto que se ha propuesto que los niveles de catecolaminas (tanto endógenas como exógenas), aumenten los requerimientos de propofol $y$, en consecuencia, aumente el riesgo de desarrollar PRIS[21].

\section{Presentación Clínica}

Debido a la fisiopatología subyacente (lipólisis con inhibición de la oxidación de ácidos grasos, disfunción mitocondrial y medicamentos concurrentes) la presentación clínica involucra el compromiso de múltiples sistemas. Los sistemas más frecuentemente involucrados son el cardiovascular, hepático, esquelético, renal y metabólico[7] (Tabla 2).

\section{Cardiovascular}

Las manifestaciones cardiacas principalmente son arritmias (particularmente de tipo Brugada), ensanchamiento del QRS y arritmias ventriculares. Posteriormente, la evolución natural involucra bradicardia refractaria a tratamiento, shock cardiogénico y asistolia[7]. 
Tabla 2. Síntomas comunes del PRIS

\begin{tabular}{|c|c|}
\hline Cardiovascular & $\begin{array}{l}\text { 1. Arritmias } 60-80 \% \\
\text { - (especialmente Brugada Tipo I) } \\
\text { 2. Falla cardiaca } 20-30 \% \\
\text { 3. Hipotensión } 20-30 \%\end{array}$ \\
\hline Músculoesquelético & $\begin{array}{l}\text { 1. Rabdomiolisis } 40-60 \% \\
\text { 2. Miopatía }\end{array}$ \\
\hline Metabólico & $\begin{array}{l}\text { 1. Acidosis metabólica con Anion Gap aumentado } 80 \% \\
\text { 2. Hipertrigliceridemia } 20-40 \%\end{array}$ \\
\hline Renal & 1. Injuria renal aguda $40 \%$ \\
\hline Hepático & 1. Pruebas hepáticas alteradas $10-20 \%$ \\
\hline Otros & 1. Fiebre $10-40 \%$ \\
\hline
\end{tabular}

\section{Músculoesquelético}

En un principio se observa una miopatía que frecuentemente progresa a una rabdomiólisis. Asociado a esto se puede observar hiperkalemia e injuria renal aguda por destrucción muscular[7].

\section{Metabólico}

Es característica la acidosis con anion gap aumentado. Esta se produciría por un aumento de la producción de ácido láctico por la destrucción muscular, inhibición de la fosforilación oxidativa, privilegiando el metabolismo anaerobio[22].

Frecuentemente existirá hiperkalemia debido a la liberación de potasio por destrucción muscular. Sumado a esto la acidosis favorecerá el intercambio de $\mathrm{H}+$ del extracelular por $\mathrm{K}+$ en el intracelular. A nivel hepático se produce elevación de enzimas, esteatosis y hepatomegalia. Es frecuente ver hipertrigliceridemia pero no se ha dilucidado si es debido a una lipólisis aumentada o al hecho de que el propofol se administra en una formulación lipídica al 1\%[7].

Como se puede observar, las manifestaciones del PRIS son vagas y se pueden presentar en una serie de cuadros que afectan al paciente crítico. Debido a esto se debería considerar el diagnóstico en pacientes que han recibido dosis altas de propofol (> $4 \mathrm{mg} / \mathrm{kg} / \mathrm{h}$ ) o por un tiempo prolongado (> $48 \mathrm{~h})[1],[7]$.

\section{Prevención}

\section{Screening}

Un estudio reciente antes-después publicado por Schroeppel et al., demostró que los pacientes que desarrollaron PRIS tenían una CK significativamente superior a los controles. En una segunda fase se creó un protocolo de screening, en el cual se detenía la infusión de propofol con un punto de corte de CK de $5.000 \mathrm{U} / \mathrm{L}$. En un seguimiento de 72 meses, el cual incluyó a 1.038 pacientes, ninguno desarrolló PRIS[24].

\section{Intervenciones protectoras}

Debido a que el principal factor de riesgo para el desarrollo del síndrome es la dosis y tiempo de administración del propofol, se debe mantener la infusión por un máximo de $48 \mathrm{~h}$ y no superar los $4 \mathrm{mg} / \mathrm{kg} / \mathrm{h}$ [17].

En el paciente críticamente enfermo, la depleción de carbohidratos promueve la movilización de reservas grasas y la lipólisis. Esto promueve un aumento de los ácidos grasos circulantes y podría predisponer al PRIS[25]. El aporte de carbohidratos en dosis de 6-8 $\mathrm{mg} / \mathrm{kg} / \mathrm{min}$ permitiría favorecer el metabolismo de estos por sobre el de los ácidos grasos, previniendo así el desarrollo del síndrome[13],[25].

\section{Tratamiento}

La mayoría del conocimiento del manejo del PRIS proviene de reportes de casos y estudios en animales. Además, no se ha desarrollado un antídoto o terapia específica y la mayoría de los pacientes en los reportes ha fallecido. Es por esto por lo que el tratamiento del PRIS es meramente de soporte e involucra el manejo de las manifestaciones clínicas.

En un paciente con alta sospecha de PRIS la primera medida a tomar es la suspensión inmediata de la infusión de propofol[1],[7]. Las manifestaciones cardiovasculares son las más graves y deben ser manejadas adecuadamente. El shock cardiogé- 
nico debe ser manejado de acuerdo con las guías vigentes[26]. Debido a que el propofol inhibe los canales de calcio y posee ciertas propiedades betabloqueantes, se cree que el uso de catecolaminas y sus miméticos es menos eficaz. En este sentido medicamentos que no actúen a este nivel (milrinona, glucagón y levosimendan), serían teóricamente más efectivos[7].

La acidosis y la hiperkalemia deben ser rápidamente corregidas y la terapia de reemplazo renal considerada precozmente[27].

En los casos refractarios de PRIS se debe considerar la oxigenación por membrana extracorpórea (ECMO) como terapia de salvataje[28].

\section{Conclusión}

El síndrome de infusión por propofol es una complicación rara pero altamente letal del uso de la droga. La manifestación clínica es altamente heterogénea y no existen criterios diagnósticos unificados. Los síntomas que se observan en mayor proporción son las arritmias cardiacas de difícil tratamiento, rabdomiólisis y acidosis metabólica con anion gap aumentado. El principal factor de riesgo es la duración y dosis por hora de propofol. Como medida de prevención se recomienda limitar las dosis de propofol a no más de $4 \mathrm{mg} / \mathrm{kg} / \mathrm{h}$. El tratamiento se basa en la suspensión de la droga y manejo de las complicaciones.

\section{Referencias}

1. Krajcová $A$, Waldauf $P$, Andel M, Duška F, Access O. Propofol infusion syndrome: a structured review of experimental studies and 153 published case reports [Internet]. Crit Care. 2015 Nov;19(1):398.

2. Miller RD. Miller's Anesthesia. 8th ed. Elsevier; 2015. p. 3.

3. Parke TJ, Stevens JE, Rice AS, Greenaway CL, Bray RJ, Smith PJ, et al. Metabolic acidosis and fatal myocardial failure after propofol infusion in children: five case reports [Internet]. BMJ. 1992 Sep;305(6854):613-6.

4. Cremer OL, Moons KG, Bouman EA, Kruijswijk JE, de Smet AM, Kalkman CJ. Long-term propofol infusion and cardiac failure in adult head-injured patients. Lancet. 2001 Jan;357(9250):117-8.

5. Diprivan FD. (Propofol 1\%) injectable emulsion [package insert]. Lake Zurich, IL. Freseius Kabi, LLC. 2017;(April):1-54.

6. EMA. DIPRIVAN $® 1 \%$ e $2 \%$ (Propofol). Lake Drive, Ireland: Aspen Pharma; 2017.

7. Mirrakhimov AE, Voore P, Halytskyy O, Khan M, Ali AM. Propofol infusion syndrome in adults: a clinical update. Crit Care Res Pr
[Internet]. 2015;2015:260385.

8. Hwang WS, Gwak HM, Seo DW. Propofol infusion syndrome in refractory status epilepticus [Internet]. J Epilepsy Res. 2013 Jun;3(1):21-7.

9. Roberts RJ, Barletta JF, Fong JJ, Schumaker G, Kuper PJ, Papadopoulos $S$, et al. Incidence of propofol-related infusion syndrome in critically ill adults: a prospective, multicenter study. Crit Care. 2009;13(5):R169.

10. Lee JH, Ko YS, Shin HJ, Yi JH, Han SW, Kim HJ. Is there a relationship between hyperkalemia and propofol? Electrolyte Blood Press. 2011 Jun;9(1):27-31.

11. Uezono S, Hotta $Y$, Takakuwa Y, Ozaki M. Acquired Carnitine Deficiency: A Clinical Model for Propofol Infusion Syndrome? 2005;(4):900-11.

12. Griggs RC, Furberg C, Kawas C, Carlson M. Propofol-Related Infusion Syndrome Heralding a Mitochondrial Disease. Case Rep Neurol. 2014;82(1):1192-3.

13. Safety D, Universit VF. Fodale V, La Monaca E: Propofol infusion syndrome: An overview of a perplexing disease. 2016;(March).

14. Branca D, Roberti MS, Lorenzin $P$, Vincenti E, Scutari G. Influence of the anesthetic 2,6-diisopro- pylphenol on the oxidative phosphorylation of isolated rat liver mitochondria. Biochem Pharmacol. 1991 Jun;42(1):87-90.

15. Vanlander AV, Okun JG, de Jaeger A, Smet J, De Latter E, De Paepe B, et al. Possible pathogenic mechanism of propofol infusion syndrome involves coenzyme q. Anesthesiology. 2015 Feb;122(2):343-52.

16. Rinaldo $P$, Cowan TM, Matern D. Acylcarnitine profile analysis. Genet Med. 2008 Feb;10(2):1516.

17. Kam PC, Cardone D. Propofol infusion syndrome. Anaesthesia. 2007 Jul;62(7):690-701.

18. Cree MG, Wolfe RR. Postburn trauma insulin resistance and fat metabolism. Am J Physiol Metab [Internet]. 2008;294(1):E1-9.

19. Zhou W, Fontenot HJ, Wang SN, Kennedy RH. Propofol-induced alterations in myocardial betaadrenoceptor binding and responsiveness. Anesth Analg. 1999 Sep;89(3):604-8.

20. Zhou W, Fontenot HJ, Liu S, Kennedy RH. Modulation of cardiac calcium channels by propofol. Anesthesiology. 1997 Mar;86(3):670-5. PMID:9066334

21. Vasile B, Rasulo F, Candiani A, Latronico N. The pathophysiolo- 
gy of propofol infusion syndrome: a simple name for a complex syndrome. Intensive Care Med. 2003 Sep;29(9):1417-25.

22. Mitch W, Goldberg A. Etiology and therapeutic approach to elevated lactate. Mayo Clin Proc. 1995;335(25):359-71.

23. Myburgh JA, Upton RN, Grant C, Martinez A. Epinephrine, norepinephrine and dopamine infusions decrease propofol concentrations during continuous propofol infusion in an ovine model. Intensive Care Med. 2001 Jan;27(1):276-82.

24. Schroeppel TJ, Fabian TC, Cle- ment LP, Fischer PE, Magnotti LJ, Sharpe JP, et al. Propofol infusion syndrome: A lethal condition in critically injured patients eliminated by a simple screening protocol. Injury [Internet]. Elsevier Ltd; 2014.

25. Wolf $A$, Weir $P$, Segar $P$, Stone J, Shield J. Impaired fatty acid oxidation in propofol infusion syndrome. Lancet. 2001 Feb;357(9256):606-7.

26. Van Diepen S, Katz JN, Albert NM, Henry TD, Jacobs AK, Kapur NK, et al. Contemporary Management of Cardiogenic Shock: A Scientific Statement from the American
Heart Association. Vol. 136, Circulation. 2017. 232-268 p

27. Casserly B, O'Mahony E, Timm EG, Haqqie S, Eisele G, Urizar R. Propofol infusion syndrome: an unusual cause of renal failure. Am J Kidney Dis. 2004 Dec;44(6):e98-101.

28. Mayette M, Gonda J, Hsu JL, Mihm FG. Propofol infusion syndrome resuscitation with extracorporeal life support: A case report and review of the literature. Ann Intensive Care [Internet] [Available from: Annals of Intensive Care]. Ann Intensive Care. 2013;3(1):1-6. 